\title{
Validation of the prognostic impact of the new tumor-node-metastasis clinical staging in patients with gastric cancer
}

\author{
Etsuro Bando $^{1} \cdot$ Rie Makuuchi $^{1} \cdot$ Tomoyuki Irino $^{1} \cdot$ Yutaka Tanizawa $^{1} \cdot$ Taiichi Kawamura $^{1} \cdot$ Masanori Terashima $^{1} \mathbb{D}$
}

Received: 18 September 2017 / Accepted: 16 January 2018 / Published online: 22 January 2018

(c) The International Gastric Cancer Association and The Japanese Gastric Cancer Association 2018

\begin{abstract}
Background In the 8th edition of the tumor-node-metastasis (TNM) classification, the gastric cancer staging system includes two classifications: the clinical stage (cStage) and the postoperative pathologic stage. However, the correlation between the new cStage and overall survival has not been studied. Moreover, clinical N $(\mathrm{cN})$ grade analysis is not included in the new clinical staging system. This study validated the prognostic value of cStage in the 8th edition of the TNM classification and the significance of $\mathrm{N}$ classification for pretreatment staging in gastric cancer.

Methods A total of 4374 patients with primary gastric cancer who underwent surgery at the Shizuoka Cancer Center were included. Survival analysis was conducted based on the newly proposed cStage criteria of the TNM 8th edition. Prognostic accuracy was evaluated using Harrell's concordance index (C-index).

Results The five-year survival rates according to cStage were as follows: cStageI, 91.2\%, cStageIIA: 75.1\%, cStageIIB: 57.7\%, cStageIII: $43.2 \%$, cStageIVA: $31.6 \%$, and cStageIVB: $7.7 \%$. Significant differences were observed among all stages $(P<0.001)$. The cStage C-index was 0.802. Meanwhile, the five-year survival rates based on $\mathrm{cN}$ were as follows: $\mathrm{cN} 0$ : $83.7 \%, \mathrm{cN} 1: 57.2 \%, \mathrm{cN} 2: 42.4 \%, \mathrm{cN} 3 \mathrm{a}: 22.1 \%$, and cN3b: $0.0 \%$. Significant differences were also observed among all cN grades $(P<0.001)$.

Conclusions The cStage of the 8th edition of the TNM classification has a good capability to predict prognosis; thus, it may be a useful indicator for selecting appropriate gastric cancer treatment modalities. In addition, $\mathrm{cN}$ can be included in developing a more precise clinical staging of gastric cancer.
\end{abstract}

Keywords Stomach neoplasms $\cdot$ Neoplasm staging $\cdot$ Survival

\section{Introduction}

Malignant tumors had been classified into clinical stages based on the TNM classification defined by the American Joint Committee on cancer (AJCC) [1] and the Union for International Cancer Control (UICC) [2]. Up till the 7th edition [3], the classification for gastric cancer had largely

All authors contributed equally to this work.

Electronic supplementary material The online version of this article (https://doi.org/10.1007/s10120-018-0799-9) contains supplementary material, which is available to authorized users.

Masanori Terashima

m.terashima@scchr.jp

1 Division of Gastric Surgery, Shizuoka Cancer Center, Shimonagakubo 1007, Nagaizumichou, Suntougun, Shizuoka 411-0934, Japan been based on pathologic data obtained from the resected specimens (pStages), which meant that the clinical stage served primarily as a prognostic determinant based on which postoperative treatment strategies could be chosen. In this aspect, the 8th edition incorporated a new pathologic staging system proposed by the International Gastric Cancer Association which was designed based on data from 59 institutions in 15 countries [4].

Given that an increasing number of gastric cancer patients are treated by neoadjuvant chemotherapy (NAC) especially in the Western countries [5, 6], the 8th edition declared that, in addition to the pStages, a clinical stage classification (cStages) be established based on evidence acquired before treatment so that the a broad range of treatment strategies including preoperative therapies can be selected and evaluated. However, reports on the correlation between TNM classification and survival have been mostly based on the pStages obtained after surgery [7-10], and only a few 
attempts to evaluate clinical staging prior to surgery had been made [11, 12]. The AJCC Cancer Staging Manual [1] provides survival curves that were used as a basis of the cStage in the 8th edition, but does not provide detailed data for the cT or cN categories. Statistical evaluation of the survival curves for each cStage and the basis for the subclassification of Stage IV into Stages IVA and IVB are also lacking.

In the current study, statistical assessments were conducted to evaluate appropriateness of the newly defined cStages as a tool for predicting gastric cancer prognosis. In addition, the significance of $\mathrm{cN}$ classification based on the number of lymph nodes that are suspected to harbor metastasis was investigated.

\section{Materials and methods}

\section{Patient population}

Among the 4534 patients with primary gastric cancer who underwent surgery between October 2002 and November 2016 in the Division of Gastric Surgery at Shizuoka Cancer Center, 160 patients who received preoperative chemotherapy were excluded and the remaining 4374 patients were classified into the $\mathrm{cT}$ categories, $\mathrm{cN}$ categories and cStages according to the 8th edition of the TNM classification for comparison of the survival curves. Moreover, this study included patients with Siewert type III tumors, for which, the staging for gastric cancer was applied for the first time since the implementation of the 8th edition. Siewert Type III EGJ tumors were observed in 66 patients $(1.5 \%)$.

This study was approved by the Research Ethics Committee at Shizuoka Cancer Center (No. 29-J6-29-1-3).

\section{Criteria for determining preoperative clinical tumor progression}

The preoperative invasion depth (cT), lymph node metastasis $(\mathrm{cN})$, and distant metastasis $(\mathrm{cM})$ were determined in accordance with a previously published report [12]. A brief summary is presented below.

With regard to the endoscopic diagnosis of invasion depth, based on the diagnostic criteria defined by Ono and Abe et al. [13, 14], the macroscopic type was the most important factor affecting invasion depth. Other influencing factors included changes in the color of the gastric mucosa, abnormal folds of the gastric wall, tumor size, tumor thickness, ulceration depth, or the degree of gastric wall hardening. Contrast upper gastrointestinal radiography was performed when possible. The invasion depth was determined based on the gastric wall thickness, poor extension of the gastric wall, gastric wall hardening, and tumor size, which were proposed as important factors by Egashira et al. [15].
The findings from a multi-detector computed tomography (MDCT) scan were also important in terms of diagnosing serosal invasion (cT4) [16]. When cT4b was strongly suspected on CT, ultrasonography was additionally performed to verify the state of infiltration [17]. The $\mathrm{cT}$ was determined based on a comprehensive assessment using the various aforementioned diagnostic modalities. Endoscopic ultrasonography was not routinely performed.

Lymph node metastasis was diagnosed based on lymph node length, aggregation, and contrast imaging pattern, which were determined via an MDCT [18].

The MDCT was also used to determine cM.

The preoperative diagnosis was determined by a multidisciplinary team conference comprising surgeon endoscopists, radiologists, and oncologists.

\section{Policies for selecting treatment strategy}

The therapeutic strategy and surgical procedures were determined based on the preoperative cStage diagnosis and other relevant clinical findings. The extent of lymphadenectomy and chemotherapy (both in the postoperative adjuvant setting and advanced/metastatic setting) were determined by Japanese guidelines [19] or protocol of participated clinical trials.

The standard therapy for cM1 was chemotherapy; however, in patients with liver metastasis, hepatectomy was also considered when magnetic resonance imaging using gadolinium-ethoxybenzyl-diethylenetriaminepentaacetic acid shows $\leq 3$ metastatic tumors [20, 21]. Even in StageIV, we performed palliative gastrectomy or bypass surgery, if patients had urgent complaints including in insufficient oral intake or active bleeding from the gastric tumor.

\section{Statistical analysis}

The survival analysis was performed using the Kaplan-Meier product-limit method and log-rank tests. When $P<0.05$, the difference was considered statistically significant. Harrell's concordance index (C-index) derived from the time-dependent receiver-operating characteristic (ROC) curve, which is used to subjectively evaluate the information on prognosis prediction, was used [22]. The $\mathrm{C}$-index values ranged from 0.5 (a predictive capability equivalent to that of a coin toss) to 1.0 (perfect prognostic predictive capability). Values for area under the curve (AUC) derived from the usual ROC curve analysis also range from 0.5 to 1.0 : a value of 0.7 to 0.8 indicates a moderate predictive capability, while a value $\geq 0.8$ indicates an excellent predictive capability [23]. Similarly, a C-index $\geq 0.8$ can be interpreted as having an excellent prognostic predictive capability [24]. The statistical analysis was performed using SPSS version 18.0 Base, Regression and Advanced software programs (SPSS Inc., 
Chicago, IL, USA) and STATA/SE 14.2 (StataCorp., College Station, TX, USA).

\section{Results}

\section{Pre-operative tumor progression}

Table 1 shows the distributions of $\mathrm{cT}, \mathrm{cN}$, and $\mathrm{cM}$ based on the diagnostic criteria used in the present study. With regard to cT, cT 1 accounted for approximately $50 \%$ of the patients. In patients with advanced cancer, cT4a was observed most frequently. Meanwhile, in cN, cN0 accounted for approximately $70 \%$ of the patients. Distant metastasis was identified prior to treatment in approximately $5 \%$ of the subjects.

Two-thousand nine-hundred eighty-four $(68.2 \%)$ patients were males and remaining $1390(31.8 \%)$ were females. The median age of this cohort was 67 years. The most frequent location of the tumor was the middle third of the stomach. With regard to the histologic type [25] (i.e., pathological tissue type determined via endoscopic biopsy), the ratio of differentiated type to undifferentiated type was approximately $1: 1$. In terms of macroscopic type, approximately $60 \%$ of patients had early stage cancer. Meanwhile, among patients with advanced cancer, the most frequently diagnosed was

Table 1 Preoperative tumor progression according to the 8th edition of the AJCC/UICC TNM classification

\begin{tabular}{lll} 
cT & & \\
T1a (mucosa) & 1019 & $(23.3 \%)$ \\
T1b (submucosa) & 1180 & $(27.0 \%)$ \\
T2 (muscularis propria) & 536 & $(12.3 \%)$ \\
T3 (subserosa) & 298 & $(6.8 \%)$ \\
T4a (serosa exposed) & 1218 & $(27.8 \%)$ \\
T4b (infiltrating to adjacent organ) & 123 & $(2.8 \%)$ \\
cN & & \\
N0 (no metastases in regional node) & 3186 & $(72.8 \%)$ \\
N1 (1-2 positive in regional node) & 418 & $(9.6 \%)$ \\
N2 (3-6 positive in regional node) & 491 & $(11.2 \%)$ \\
N3a (7-15 positive in regional node) & 244 & $(5.6 \%)$ \\
N3b (16 or more positive in regional node) & 35 & $(0.8 \%)$ \\
cM & & \\
M0 (no distant metastasis) & 4167 & $(95.3 \%)$ \\
M1 (distant metastasis) & 207 & $(4.7 \%)$ \\
cStage & & \\
I & 2546 & $(58.2 \%)$ \\
IIA & 180 & $(4.1 \%)$ \\
IIB & 591 & $(13.5 \%)$ \\
III & 768 & $(17.6 \%)$ \\
IVA & 82 & $(1.9 \%)$ \\
IVB & 207 & $(4.7 \%)$ \\
\hline
\end{tabular}

invasive phenotype type 3. Surgery for 151 patients (3.5\%) was conducted as a palliative care (Table 2).

Table 3 shows the surgical procedures performed among the patients. Approximately $95 \%$ of the patients underwent gastrectomy. Approximately $40 \%$ of the patients underwent standard D2 or extended D2 + lymphadenectomy.

Table 4 shows the final distributions of pStage: pT1 was observed in 55\%, pT3/4 in 30\%, pN positive in $40 \%$, and pStage IV in $17 \%$ of patients. R1 and R2 surgery

Table 2 Preoperative tumor characteristics and the aim of surgery

\begin{tabular}{lrr}
\hline Location & & \\
Lower third & 1259 & $(28.8 \%)$ \\
Middle third & 1980 & $(45.2 \%)$ \\
Upper third & 834 & $(19.1 \%)$ \\
Entire stomach & 235 & $(5.4 \%)$ \\
EGJ & 66 & $(1.5 \%)$ \\
Histologic type (biopsied materials) & & \\
Well differentiated type & 1036 & $(23.7 \%)$ \\
Moderately differentiated type & 1251 & $(28.6 \%)$ \\
Undifferentiated type & 2087 & $(47.7 \%)$ \\
Macroscopic type & & \\
Type 0 & 2551 & $(58.4 \%)$ \\
Type 1 & 130 & $(3.0 \%)$ \\
Type 2 & 614 & $(14.0 \%)$ \\
Type 3 & 788 & $(18.0 \%)$ \\
Type 4 & 291 & $(6.7 \%)$ \\
The aim of surgery & & \\
Cure & 4223 & $(96.5 \%)$ \\
Palliation & 151 & $(3.5 \%)$ \\
\hline
\end{tabular}

EGJ esophagogastric junction tumor

Table 3 Surgical procedure

\begin{tabular}{lll}
\hline Operative method & & \\
Total & 1001 & $(22.9 \%)$ \\
Distal & 2556 & $(58.4 \%)$ \\
PPG & 346 & $(7.9 \%)$ \\
Proximal & 198 & $(4.5 \%)$ \\
Bypass & 137 & $(3.1 \%)$ \\
Simple laparotomy & 45 & $(1.0 \%)$ \\
Staging laparoscopy & 91 & $(2.1 \%)$ \\
Lymphadenectomy (/4101 resected cases) & \\
D1, D1 + & 2458 & $(59.9 \%)$ \\
D2, D2+ & 1643 & $(40.1 \%)$ \\
Approach & & \\
Open & 3397 & $(77.7 \%)$ \\
Laparoscopic & 746 & $(17.1 \%)$ \\
Robot-assisted & 231 & $(5.3 \%)$ \\
\hline
\end{tabular}

$P P G$ pylorus-preserved gastrctomy 
Table 4 Postoperative tumor progression according to the 8th edition of the AJCC/UICC TNM classification

\begin{tabular}{|c|c|c|}
\hline \multicolumn{3}{|l|}{$\mathrm{pT}(N=4101$ resected cases $)$} \\
\hline T1a (mucosa) & 942 & $(23.0 \%)$ \\
\hline T1b (submucosa) & 1319 & $(32.2 \%)$ \\
\hline T2 (muscularis propria) & 432 & $(10.5 \%)$ \\
\hline T3 (subserosa) & 647 & $(15.8 \%)$ \\
\hline T4a (serosa-exposed) & 691 & $(16.8 \%)$ \\
\hline T4b (infiltrating to adjacent organ) & 70 & $(1.7 \%)$ \\
\hline \multicolumn{3}{|l|}{$\mathrm{pN}(N=4101$ resected cases $)$} \\
\hline N0 (no metastases in regional node) & 2417 & $(58.9 \%)$ \\
\hline N1 (1-2 positive in regional node) & 596 & $(14.5 \%)$ \\
\hline N2 (3-6 positive in regional node) & 405 & $(9.9 \%)$ \\
\hline N3a (7-15 positive in regional node) & 372 & $(9.1 \%)$ \\
\hline $\mathrm{N} 3 \mathrm{~b}$ (16 or more positive in regional node) & 311 & $(7.6 \%)$ \\
\hline \multicolumn{3}{|l|}{$\mathrm{pM}$} \\
\hline M0 (no distant metastasis) & 3631 & $(83.0 \%)$ \\
\hline M1 (distant metastasis) & 743 & $(17.0 \%)$ \\
\hline \multicolumn{3}{|l|}{ pStage } \\
\hline IA & 1925 & $(44.0 \%)$ \\
\hline IB & 436 & $(10.0 \%)$ \\
\hline IIA & 377 & $(8.6 \%)$ \\
\hline IIB & 312 & $(7.1 \%)$ \\
\hline IIIA & 289 & $(6.6 \%)$ \\
\hline IIIB & 202 & $(4.6 \%)$ \\
\hline IIIC & 90 & $(2.1 \%)$ \\
\hline IV & 743 & $(17.0 \%)$ \\
\hline \multicolumn{3}{|l|}{ Residual tumor $=\mathrm{R}$} \\
\hline 0 & 3663 & $(83.7 \%)$ \\
\hline 1 & 253 & $(5.8 \%)$ \\
\hline 2 & 458 & $(10.5 \%)$ \\
\hline
\end{tabular}

were performed in $253(5.8 \%)$ and 458 (10.5\%) patients, respectively.

\section{Survival outcome according to preoperative clinical tumor depth of invasion (cT)}

The survival curves for the six staging-related cT groups are presented in Fig. 1. In each tumor depth group, the five-year survival rates were as follows: cT1a: $92.9 \%$, cT1b: $90.7 \%$, cT2: $82.4 \%$, cT3: $69.7 \%$, cT4a: $39.2 \%$, and cT4b: $21.5 \%$. A significant difference in overall survival was observed among all groups except between cT1a and cT1b $(P<0.001$; cT1a vs. cT1b, $P=0.015$ ).

\section{Survival outcome according to preoperative clinical nodal involvement (cN)}

Figure 2 shows the survival curves according to preoperative grade of lymph node metastasis. In each grade, the five-year survival rates were as follows: $\mathrm{cN} 0: 83.7 \%, \mathrm{cN} 1: 57.2 \%$,

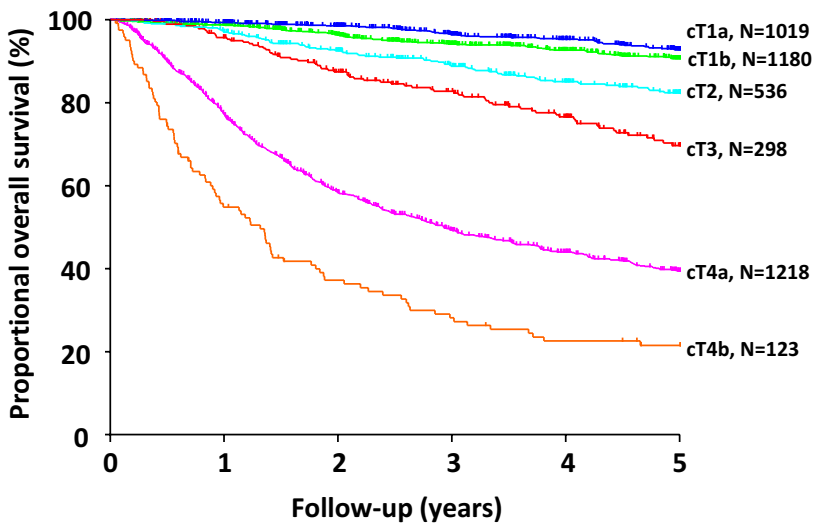

Fig. 1 Kaplan-Meier survival curves of overall survival of patients with gastric cancer stratified into six clinical depths of tumor invasion (cT; T1a-T1b-T2-T3-T4a-T4b) according to the 8th Edition of the TNM classification

cN2: $42.4 \%$, cN3a: $22.1 \%$, and cN3b: $0.0 \%$. A significant difference in overall survival was observed among all groups $(P<0.001)$.

\section{Survival outcome according to the newly established clinical stage in the 8th edition TNM}

The survival curves by cStage classification determined according to $\mathrm{cT}$, $\mathrm{cN}$, and $\mathrm{cM}$ are presented in Fig. 3. The five-year survival rates were as follows: cStageI $(N=2546)$ : $91.2 \%$, cStageIIA $(N=180): 75.2 \%$, cStageIIB $(N=591)$ : $59.3 \%$, cStageIII $(N=768): 43.4 \%$, cStageIVA $(N=82)$ : $28.6 \%$, and cStageIVB $(N=207): 6.9 \%$. A significant difference in overall survival was observed among all stages $(P<0.001)$. In the present 8 th edition, the Harrell's C-index for cStage was 0.802 (95\% CI 0.791-0.815).

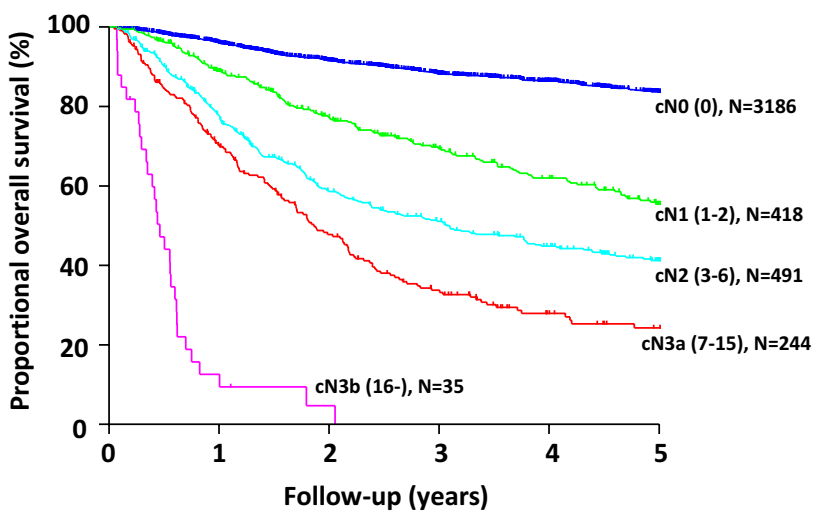

Fig. 2 Kaplan-Meier survival curves of overall survival of patients with gastric cancer stratified into five groups by clinical nodal involvement (cN; N0-N1-N2-N3a-N3b) according to the 8th Edition of the TNM classification 


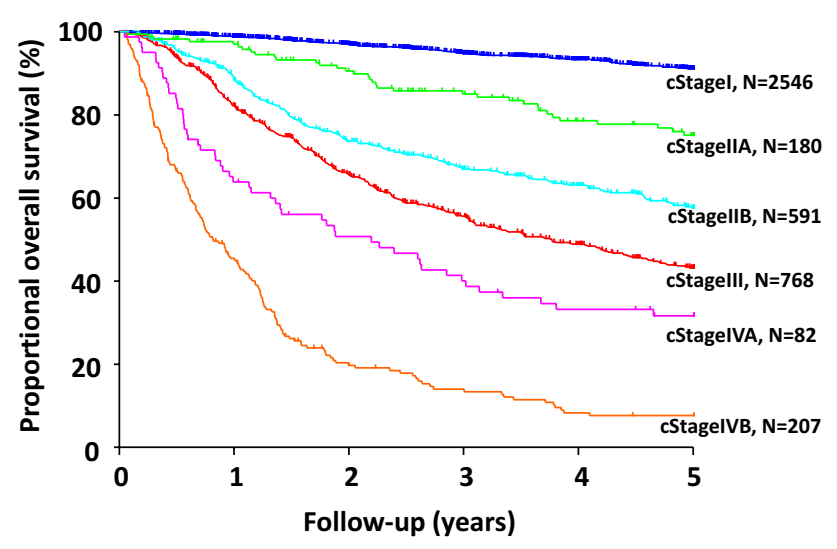

Fig. 3 Kaplan-Meier survival curves of overall survival of patients with gastric cancer stratified into six clinical stages (cStage) according to the 8th edition of the TNM classification

\section{Discussion}

We have previously published the prognostic impact of clinical staging of gastric cancer based on the 7th TNM [12]. Also in this study, our results showed the excellent prognostic impact of the cStage proposed in the 8th edition of the TNM classification. Furthermore, we also demonstrated the prognostic capability of the $\mathrm{cN}$ grade.

Although the roles of cStage and pStage differ, the same classification was used in both clinical and pathological staging until the 7 th edition. However, in the 8th edition, they were divided according to their original roles.

As the cStage and pStage categories are different in the 8th edition, direct comparison of stage-concordance rates is impossible. Therefore, we separately evaluated the concordance rate in $\mathrm{cT}$ or $\mathrm{cN}$.

Regarding the $\mathrm{T}$ categories, the concordance rates were $25.1 \%$ in $\mathrm{T} 2,36.1 \%$ in $\mathrm{T} 3$, and $57.6 \%$ in $\mathrm{T} 4$ in our study (Supplemental Table 1). In a multicenter prospective study (JCOG1302), in which prospectively recorded clinical diagnoses (cT and $\mathrm{cN}$ categories) were compared with postoperative pathological diagnoses (pT and $\mathrm{pN}$ categories) [26], the concordance rates were $28.9,38.2$, and $55.9 \%$ in $\mathrm{T} 2$, $\mathrm{T} 3$, and T4, respectively. The concordance rate of cT-pT was relatively high in T4. However, it was about $40 \%$ in T3 and lower than $30 \%$ in $\mathrm{T} 2$, respectively. These data were considered unsatisfactory. The AJCC cancer staging manual [1] recommends cT diagnosis via endoscopic ultrasound (EUS), although its usefulness for determining the depth of advanced gastric cancer is not mentioned. Razavi et al. [27] reported the value of EUS for determining depth of gastric cancer in 106 patients, including those in advanced stages. However, the overall diagnostic accuracy rate was $70 \%$ or lower and the sensitivity of T4 diagnosis was 48\%. Most Japanese studies on the usefulness of EUS aimed to determine the indication for endoscopic submucosal dissection and, therefore, mostly covered early gastric cancer patients [28, 29]. Evidence on the usefulness of EUS for determining cancer depth in T2-T4 cases remains limited. Even if EUS was performed in all patients, satisfactory improvement of concordance rate could have been difficult.

Regarding N category, the concordance rates were $28.5 \%$ in N1, 22.7\% in N2, and $72.3 \%$ in N3 in our study (Supplemental Table 1), with a cN-positive/pN-positive concordance rate of $77.5 \%$ in JCOG1302 study [26].

We also compared the $\mathrm{cT} / \mathrm{cP}$ and $\mathrm{cN} / \mathrm{pN}$ from the viewpoint of survival. There were not so much differences between cT and pT in T2/3/4 (Supplemental Fig. 1a/b/c). However, there were obvious much differences between $\mathrm{cN}$ and $\mathrm{pN}$ in any $\mathrm{cN}$ grading (Supplemental Fig. 2a/b/c).

Because attaining satisfactory $\mathrm{cT} / \mathrm{pT}$ and $\mathrm{cN} / \mathrm{pN}$ concordance rate, and getting same survival outcome in $\mathrm{cN} / \mathrm{pN}$, are difficult, establishing a reliable original cStage apart from pStage is important.

We showed that the survival curves were clearly separated even when six categories of cT1a to cT4b were used.

In the 8 th edition, the degree of $\mathrm{cN}$ positivity is not used in cancer staging. This is probably due to a lack of data in counting the number of metastatic lymph nodes on CT-scan.

With our data, the survival curves were clearly separated using the five classes of $\mathrm{cN} 0-\mathrm{cN} 3 \mathrm{~b}$. The survival curves of the three (cN0-N1-N2/3) and four (cN0-N1-N2-N3) $\mathrm{cN}$ groups were also arbitrary investigated (Supplemental Fig. 3a/b). In a practical sense, the use of four or five (cN0N1-N2-N3a-N3b) cN groups is probably too complicated. We also evaluated the survival outcome of cStage IIA/III/ IVA according to three $\mathrm{cN}$ group (Supplemental Fig. $4 \mathrm{a} / \mathrm{b} / \mathrm{c}$ ). The results revealed $\mathrm{cN}$-based survival curves that were not separated in cStage IIA/IVA, although a clear separation was shown in cStage III $(P<0.001)$.

So, we propose classifying $\mathrm{cN} 2 \sim \mathrm{cN} 3 \mathrm{~b}$ into one group and splitting cStage III into cStages IIIA (cT3T4/cN1) and cIIIB (cT3T4/cN2/3).

The survival curves produced by our center and the US National Cancer Database (NCDB) are presented in the AJCC Cancer Staging Manual [1]. The survival curves are clearly separated in both cohorts. The new cStage is considered reliable and easy to use in practice. However, more precise classification may be beneficial in clinical practice.

In the Japanese guidelines [19], the extent of lymphadenectomy differs between cT1 and cT2. Moreover, in the National Comprehensive Cancer Network guidelines [5], preoperative chemotherapy or preoperative chemoradiation is recommended in some cases of cT2. Because the cStage is an index used to select appropriate treatment modalities, we believe that establishing cT1 and cT2 subclasses for cStageI would be beneficial. We examined overall survival after dividing cStageI into IA (T1N0) and IB (T2N0). The 
five-year survival rate was $92.4 \%$ in cStage IA, significantly higher than that in cStage IB $(83.7 \%, P<0.001)$ (Supplemental Fig. 5).

Regarding neoadjuvant chemotherapy (NAC), the JCOG 1302 trial proposed the criterion "cT3/T4 and cN1/N2/N3" disease based on the adequate sensitivity of pStage III and low contamination rate of pStage I [26]. This stage is incidentally concordant to cStage III in the current TNM staging. In our survival data, the treatment outcome of cStage III was unsatisfactory, with $43.2 \%$ at five years. In contrast, NAC is recommended in a broader population of advancedstage gastric cancer in the Western countries. Consequently, however, a substantial number of patients with pStageI cancer for whom the adjuvant treatment is unnecessary will have to receive the cytotoxic agents. Attempts to create precise clinical staging as we did in this study might be useful to establish more strict criteria for NAC even for patients in the Western countries.

The AJCC recently announced guidelines for evaluating the published statistical prediction models that will be endorsed for clinical use [30]. As a customized tool for predicting prognosis in gastric cancer, Kattan et al. [31] established a gastric cancer nomogram. According to the AJCC [1], the pretreatment clinical factors that should be collected in the future include the number of suspicious nodes, the site of such lymph nodes, invasion depth, tumor location, tumor size, sites of distant metastasis, and serum tumor markers. The data from the present study may be of help, in part, for the revision of the new TNM classification.

The present study has several limitations. The data were collected retrospectively in a single institution. However, diagnoses for $\mathrm{cT}, \mathrm{cN}$, and $\mathrm{cM}$ were made by endoscopists and radiologists based on uniform criteria, making the study results meaningful. Another limitation of the present study was that the data were analyzed based on the surgical database; therefore, data on more than $50 \%$ of the patients with cStageIVB were probably missing. In the future, collecting data from patients who received chemotherapy or palliative therapy as initial therapy is necessary.

In conclusion, the clinical staging of gastric cancer has a high C-index for the prediction of overall survival. Therefore, the new cStage, which is defined separately from pStage for the first time in the 8th edition of the TNM classification, can be used as a criterion for selecting the appropriate therapy for gastric cancer, including limited surgery, standard surgery, extended surgery, NAC, or chemotherapy in routine clinical practice. Moreover, new treatment strategies should be planned based on the cStage, including $\mathrm{cN}$ grade, for individualized therapy.

Funding This study was supported in part by a scientific research grant from multi-institutional trials to establish a new standard treatment of solid tumors in adults from the National Cancer Center Research and Development Fund (26-A-4) and Practical Research for Innovative Cancer Control from the Japan Agency for Medical Research and Development, AMED (15ck0106043h0002).

\section{Compliance with ethical standards}

Conflicts of Interest The authors declare that they have no conflict of interest.

Ethical standards All procedures performed were in accordance with the ethical standards of the responsible committee on human experimentation (institutional and national) and with the Helsinki Declaration of 1964 and later versions. Informed consent or an appropriate substitute was obtained from all patients prior to inclusion in the study.

\section{References}

1. Amin MB, Edge S, Greene F, Byrd DR, Brookland RK, Washington MK, et al., editors. AJCC cancer staging manual. 8th ed. New York: Springer; 2016.

2. Brierley J, Gospodarowicz M, Wittekind C, editors. TNM classification of malignant tumours. 8th ed. New York: Wiley-Blackwell; 2017.

3. Sobin L, Gospodarowicz M, Wittekind C, editors. TNM classification of malignant tumours. 7th ed. New York: Wiley-Blackwell; 2009.

4. Sano T, Coit DG, Kim HH, Roviello F, Kassab P, Wittekind C, et al. For the IGCA staging project. Proposal of a new stage grouping of gastric cancer: international Gastric Cancer Association Staging Project. Gastric Cancer. 2017;20:217-25.

5. National Comprehensive Cancer Network Guidelines. http:// www.nccn.org/professionals/physician_gls/f_guidelines.asp\#site (Accessed April 10th 2017).

6. Waddell T, Verheij M, Allum W, Cunningham D, Cervantes A, Arnold D. European Society for Medical Oncology (ESMO), European Society of Surgical Oncology (ESSO), European Society of Radiotherapy and Oncology (ESTRO). Gastric cancer: ESMO-ESSO-ESTRO clinical practice guidelines for diagnosis, treatment and follow-up. Eur J Surg Oncol. 2014;40:584-91.

7. Ahn HS, Lee HJ, Hahn S, Kim WH, Lee KU, Sano T, et al. Evaluation of the seventh American Joint Committee on Cancer/International Union Against Cancer Classification of gastric adenocarcinoma in comparison with the sixth classification. Cancer. 2010;116:5592-8.

8. Jung H, Lee HH, Song KY, Jeon HM, Park CH. Validation of the seventh edition of the American Joint Committee on Cancer TNM staging system for gastric cancer. Cancer. 2011;117:2371-8.

9. Wang J, Dang P, Raut CP, Pandalai PK, Maduekwe UN, Rattner $\mathrm{DW}$, et al. Comparison of a lymph node ratio-based staging system with the 7th AJCC system for gastric cancer: analysis of 18,043 patients from the SEER database. Ann Surg. 2012;255:478-85.

10. Marrelli D, Morgagni P, de Manzoni G, Coniglio A, Marchet A, Saragoni L, et al. Italian Research Group for Gastric Cancer (IRGGC). Prognostic value of the 7th AJCC/UICC TNM classification of noncardia gastric cancer: analysis of a large series from specialized Western centers. Ann Surg. 2012;255:486-91.

11. Park SR, Kim MJ, Ryu KW, Lee JH, Lee JS, Nam BH, et al. Prognostic value of preoperative clinical staging assessed by computed tomography in resectable gastric cancer patients: a viewpoint in the era of preoperative treatment. Ann Surg. 2010;251:428-35.

12. Bando E, Makuuchi R, Tokunaga M, Tanizawa Y, Kawamura T, Terashima M. Impact of clinical tumor-node-metastasis staging on 
survival in gastric carcinoma patients receiving surgery. Gastric Cancer. 2017;20:448-56.

13. Ono H, Yoshida Y. Endoscopic diagnosis of the depth of cancer invasion for gastric cancer. Stomach Intest. 2001;36:334-40 (in Japanese with English abstract).

14. Abe S, Oda I, Shimazu T, Kinjo T, Tada K, Sakamoto T, et al. Depth-predicting score for differentiated early gastric cancer. Gastric Cancer. 2011;14:35-40.

15. Egahira H, Baba Y, Kato H. Diagnosis of the depth of invasion of gastric cancer, diagnostic indicator of the depth of invasion by the radiological examination. Stomach Intest. 2001;36:321-33 (in Japanese with English abstract).

16. Kumano S, Murakami T, Kim T, Hori M, Iannaccone R, Nakata $\mathrm{S}$, et al. T staging of gastric cancer: role of multi-detector row $\mathrm{CT}$. Radiology. 2005;237:961-6.

17. Liao SR, Dai Y, Huo L, Yan K, Zhang L, Zhang H, et al. Transabdominal ultrasonography in preoperative staging of gastric cancer. World J Gastroenterol. 2004;10:3399-404.

18. Kim SK, Kang KW, Lee JS, Kim HK, Chang HJ, Choi JY, et al. Assessment of lymph node metastases using 18F-FDG PET in patients with advanced gastric cancer. Eur J Nucl Med Mol Imaging. 2006;33:148-55.

19. Association Japanese Gastric Cancer. Japanese gastric cancer treatment guidelines 2014 (Ver. 4). Gastric Cancer. 2017;20:1-19.

20. Koh DM, Collins DJ, Wallace T, Chau I, Riddell AM. Combining diffusion-weighted MRI with Gd-EOB-DTPA-enhanced MRI improves the detection of colorectal liver metastases. Br J Radiol. 2012;85:980-9.

21. Tatsubayashi T, Tanizawa Y, Miki Y, Tokunaga M, Bando E, Kawamura T, et al. Treatment outcomes of hepatectomy for liver metastases of gastric cancer diagnosed using contrast-enhanced magnetic resonance imaging. Gastric Cancer. 2017;20:387-93.

22. Harrell FE Jr, Lee KL, Mark DB. Multivariable prognostic models: issues in developing models, evaluating assumptions and adequacy, and measuring and reducing errors. Stat Med. 1996;15:361-87.
23. Hosmer D, Lemeshow S 2nd, editors. Applied logistic regression. New York: Wiley; 2000.

24. Harrell FE. Regression modeling strategies with applications to linear models, logistic regression, and survival analysis. New York: Springer; 2001.

25. Japanese Gastric Cancer Association. Japanese classification of gastric carcinoma: 3rd English edition. Gastric Cancer. 2011;14:101-12.

26. Fukagawa T, Katai H, Mizusawa J, Nakamura K, Sano T, Terashima M, et al. Stomach Cancer Study Group of the Japan Clinical Oncology Group. A prospective multi-institutional validity study to evaluate the accuracy of clinical diagnosis of pathological stage III gastric cancer (JCOG1302A). Gastric Cancer. 2017. https://doi.org/10.1007/s10120-017-0701-1. [Epub ahead of print].

27. Razavi SM, Khodadost M, Sohrabi M, Keshavarzi A, Zamani F, Rakhshani N, et al. Accuracy of endoscopic ultrasonography for determination of tumor invasion depth in gastric cancer. Asian Pac J Cancer Prev. 2015;16:3141-5.

28. Pei Q, Wang L, Pan J, Ling T, Lv Y, Zou X. Endoscopic ultrasonography for staging depth of invasion in early gastric cancer: a meta-analysis. J Gastroenterol Hepatol. 2015;30:1566-73.

29. Park JS, Kim H, Bang B, Kwon K, Shin Y. Accuracy of endoscopic ultrasonography for diagnosing ulcerative early gastric cancers. Medicine (Baltimore). 2016;95:e3955. https://doi. org/10.1097/MD.0000000000003955.

30. Kattan MW, Hess KR, Amin MB, Lu Y, Moons KG, Gershenwald JE, et al. American Joint Committee on Cancer acceptance criteria for inclusion of risk models for individualized prognosis in the practice of precision medicine. CA Cancer J Clin. 2016;66:370-4.

31. Kattan MW, Karpeh MS, Mazumdar M, Brennan MF. Postoperative nomogram for disease-specific survival after an R0 resection for gastric carcinoma. J Clin Oncol. 2003;21:3647-50. 